\title{
Application of Alpha Skew Truncated Cauchy Distribution in Exchange Rate Data
}

\author{
Partha Jyoti Hazarika, Subrata Chakraborty \\ Department of Statistics, Dibrugarh University, Dibrugarh, Assam, India
}

Email address:

parthajhazarika@gmail.com (P. J. Hazarika), subrata_arya@yahoo.co.in (S. Chakraborty)

\section{To cite this article:}

Partha Jyoti Hazarika, Subrata Chakraborty. Application of Alpha Skew Truncated Cauchy Distribution in Exchange Rate Data. International Journal of Statistical Distributions and Applications. Vol. 2, No. 2, 2016, pp. 22-26. doi: 10.11648/j.ijsd.20160202.12

Received: July 6, 2016; Accepted: July 9, 2016; Published: August 26, 2016

\begin{abstract}
In this article the alpha skew version of truncated Cauchy distribution using the methodology of Elal-Olivero (Alpha-skew-normal distribution. Proyecciones Journal of Mathematics. 29: 224-240, 2010) has been derived. The important distributional properties have been investigated. An application of the distribution in modeling exchange rate data from the field of finance has been presented.
\end{abstract}

Keywords: Alpha Skew Distribution, Exchange Rate, MLE

\section{Introduction}

In the successful journey of contemporary probability distributions, the importance and the value of Cauchy distribution can't be undermined in spite of its main drawback of non existence of moments and as a result the application of this distribution remains comparatively limited. To overcome this drawback, Johnson and Kotz [1] first introduced the truncated version of the Cauchy distribution and further Nadarajah and Kotz [2] studied its some of the distributional properties. The probability density function (pdf) of Truncated Cauchy Distribution (TCD), $f(x)$ and the cdf, $F(x)$ are respectively given by

$$
\begin{aligned}
& f_{T D C}(x ; A, B ; \mu, \theta)= \\
& {\left[\arctan \left(\frac{B-\mu}{\theta}\right)-\arctan \left(\frac{A-\mu}{\theta}\right)\right]^{-1}\left(1+\left(\frac{x-\mu}{\theta}\right)^{2}\right)^{-1}}
\end{aligned}
$$

and

$$
F(x)=\frac{\arctan \left(\frac{x-\mu}{\theta}\right)-\arctan \left(\frac{A-\mu}{\theta}\right)}{\arctan \left(\frac{B-\mu}{\theta}\right)-\arctan \left(\frac{A-\mu}{\theta}\right)}
$$

In particular, for $\mu=0$ and $\theta=1$ standard Cauchy distribution can be retrieved from equation (5). Chakraborty and Sarmah [3] also derived the different expression for moments and other measures and applied the distribution in equation (1) in different real life situations. But, there are many real life situations which are known to be symmetric which often exhibit departure from symmetry that is skew. The use of the symmetrical distribution distributions to model such situations is inappropriate.

Azzalini [4] first introduced the path breaking skewnormal distribution, which is nothing but a natural extension of normal distribution derived by adding an additional parameter to introduce asymmetry. The pdf of the same is given by

$$
f(x ; \lambda)=2 \varphi(x) \Phi(\lambda x) ; \quad x \in R, \lambda \in R
$$

Where, $\varphi$ and $\Phi$ are the pdf and cdf of standard normal distribution respectively and $\alpha$ is the asymmetry parameter. In the same manner the pdf of skew Cauchy distribution can be written as:

$$
f(x)=\frac{1}{\pi\left(1+x^{2}\right)}\left\{1+\frac{2}{\pi} \arctan (\alpha x)\right\} ; x \in R, \alpha \in R
$$

A lot of research works have so far been carried out to present different skew distributions starting from the underlying symmetric one to model asymmetric behavior of empirical data suitable under different situations (For a complete survey on univariate skew normal distributions see 
Chakraborty and Hazarika [5]).

Following the same methodology of Azzalni [4], Nadarajah and Kotz [6] introduced the truncated skew Cauchy distribution by taking

$$
\varphi(x)=\frac{1}{2 \arctan (h)\left(1+x^{2}\right)} ;-h \leq x \leq h
$$

and

$$
\Phi(x)=\frac{1}{2}+\frac{\arctan (x)}{2 \arctan (h)}
$$

in equation (3). The pdf of their distribution is given by

$$
f(x)=\frac{1}{2 \arctan (h)\left(1+x^{2}\right)}\left[1+\frac{\arctan (\alpha x)}{\arctan (h)}\right]
$$

The distribution in (5) can be further extended to a doubly truncated Cauchy distribution with pdf

$$
f_{T T C}(x ; A, B)=\frac{2(\arctan (\alpha x)-\arctan (A))}{[\arctan (B)-\arctan (A)]^{2}\left(1+x^{2}\right)}
$$

Huang and Chen [7] proposed a general methodology for the construction of skew-symmetric distributions starting from a symmetric (about 0) pdf $h$ (.) by introducing the concept of skew function $G($.$) , a Lebesgue measurable$ function such that, $0 \leq G(x) \leq 1$ and $G(x)+G(-x)=1 ; x \in R$, almost everywhere. A random variable $X$ is said to be skew symmetric if the pdf is of the following form

$$
f(x)=2 h(x) G(x) ; x \in R .
$$

Recently, Ashani et al. [8] derived a new truncated skew Cauchy distribution where $h(x)$ is the pdf of truncated Cauchy distribution and $G(x)$ is the cdf of uniform distribution on $[h, h]$ in equ. (7). The pdf of this new skew Cauchy distribution is given by

$$
f(x)=\frac{\alpha x+h}{2 h \arctan (h)\left(1+x^{2}\right)} ;-h \leq x \leq h, \alpha \in R .
$$

Elal-Olivero [9] introduced a new class of skew normal distribution called the Alpha-skew-normal distribution that includes both unimodal as well as bimodal normal distributions and its pdf is given by

$$
f(x ; \alpha)=\frac{\left\{(1-\alpha x)^{2}+1\right\}}{2+\alpha^{2}} \varphi(x) ;-\infty<x<\infty, \alpha \in R
$$

where, $\varphi(x)$ is the pdf of standard normal distribution. Applying this approach Harandi and Alamatsaz [10] and Hazarika and Chakraborty [11, 12] respectively investigated class of alpha skew Laplace distributions and alpha skew logistic distribution and studied their properties. Very recently in 2016, Sharafi et al. [13] introduced a generalization of alpha skew normal distribution and considered it for data modeling.

In the present study the Elal-Olivero [9] methodology has been implemented to propose the new alpha skew truncated Cauchy distribution (two sided) and investigate some of its distributional properties. An application of this distribution in modeling finance exchange rate data of the United Kingdom Pound to the United States Dollar from 1800 to 2003 has shown significant performance improvement compared to some known distributions.

\section{Alpha Skew Truncated Cauchy Distribution (ASTC)}

Definition 1. If an r. v. $X$ has the density function

$$
f_{\mathrm{ASTC}}(x ; \alpha)=\frac{\left\{(1-\alpha x)^{2}+1\right\}}{D_{(A, B)}\left(1+x^{2}\right)} ; A \leq x \leq B, \alpha \in R
$$

where,

$D_{(A, B)}=(\arctan (A)-\arctan (B))\left(\alpha^{2}-2\right)+\alpha^{2}(B-A)+\alpha \log \left[\frac{1+A^{2}}{1+B^{2}}\right]$, then $X$ is an alpha-skew-truncated Cauchy (ASTC) r. v. with parameter $\alpha, A$ and $B$ and it denoted by $X \sim \operatorname{ASTC}(\alpha)$.

In particular, for $\alpha=0$ the ASTC reduces to Standard truncated Cauchy distribution. For symmetric interval $(-h, h)$ the pdf of ASTC distribution can be written as:

$$
f(x ; \alpha)=\frac{\left\{(1-\alpha x)^{2}+1\right\}}{C\left(1+x^{2}\right)} ;-h \leq x \leq h, \alpha \in R
$$

where, $C=2-\alpha^{2}+\frac{h \alpha^{2}}{\arctan (h)}$

In figure 1 , the pdf of ASTC for different choices of the parameter $\alpha$ for fixed values of $A$ and $B$ has been plotted and in figure 2 the pdf for the symmetric version in the range $(-h, h)$ has been presented. From the figure 1 and figure 2 it can be seen that the distribution has at most two modes as in case of other alpha skew distributions like alpha normal distribution [9], alpha skew logistic [11, 12], etc.

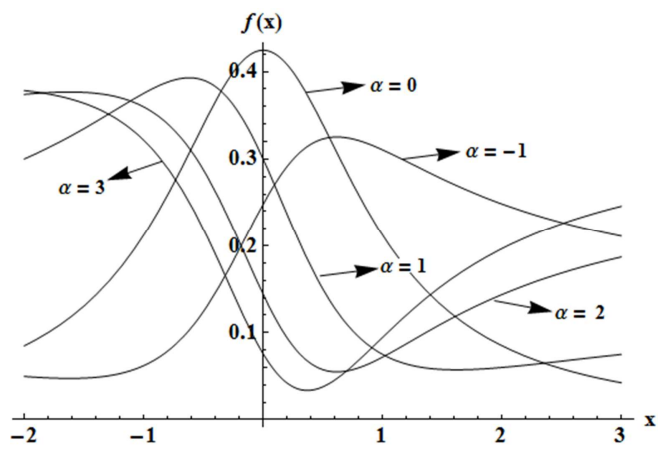

Figure 1. Shape of pdf with different choices of parameter $\alpha$ and fixed $A=-2$ and $B=3$ 


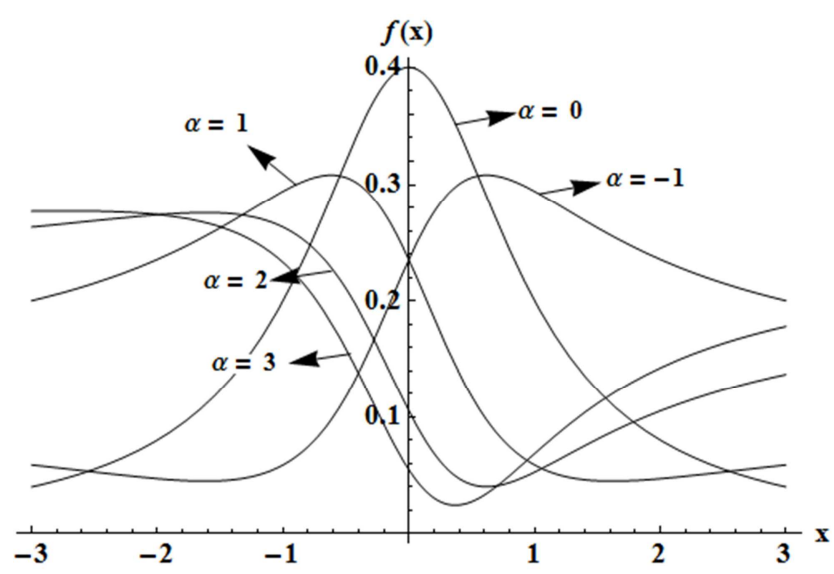

Figure 2. Shape of pdf with different choices of parameter $\alpha$ and fixed $A=-3$ and $B=3$.

Result 1: If $X \sim \operatorname{ASTC}(\alpha)$ then $-X \sim \operatorname{ASTC}(-\alpha)$

Result 2: The cdf of $X \sim \operatorname{ASTC}(\alpha)$ can be written as

$$
F(t)=P(X \leq t)=\frac{D_{(t, B)}}{D_{(A, B)}}
$$

where, $D_{(t, B)}=(\arctan (A)-\arctan (t))\left(\alpha^{2}-2\right)+\alpha^{2}(t-A)+\alpha \log \left[\frac{1+A^{2}}{1+t^{2}}\right]$.

Proof: $F(t)=P(X \leq t)=\frac{1}{D_{(A, B)}} \int_{A}^{t} \frac{\left\{(1-\alpha x)^{2}+1\right\}}{\left(1+x^{2}\right)} d x=\frac{1}{D_{(A, B)}} \int_{A}^{t} \frac{(\arctan (t)-\arctan (A))\left\{(1-\alpha x)^{2}+1\right\}}{(\arctan (t)-\arctan (A))\left(1+x^{2}\right)} d x$

$$
\begin{aligned}
& =\frac{(\arctan (t)-\arctan (A))}{D_{(A, B)}}\left[2-\int_{A}^{t} \frac{2 \alpha x}{(\arctan (t)-\arctan (A))\left(1+x^{2}\right)} d x+\int_{A}^{t} \frac{\alpha^{2} x^{2}}{(\arctan (t)-\arctan (A))\left(1+x^{2}\right)} d x\right] \\
& =\frac{(\arctan (t)-\arctan (A))}{D_{(A, B)}}\left[2-\int_{A}^{t} 2 \alpha x f_{T D C}(x ; A, t ; 0,1) d x+\int_{A}^{t} \alpha^{2} x^{2} f_{T D C}(x ; A, t ; 0,1) d x\right]
\end{aligned}
$$

The result in equation (11) follows by putting the following results in equation (12)

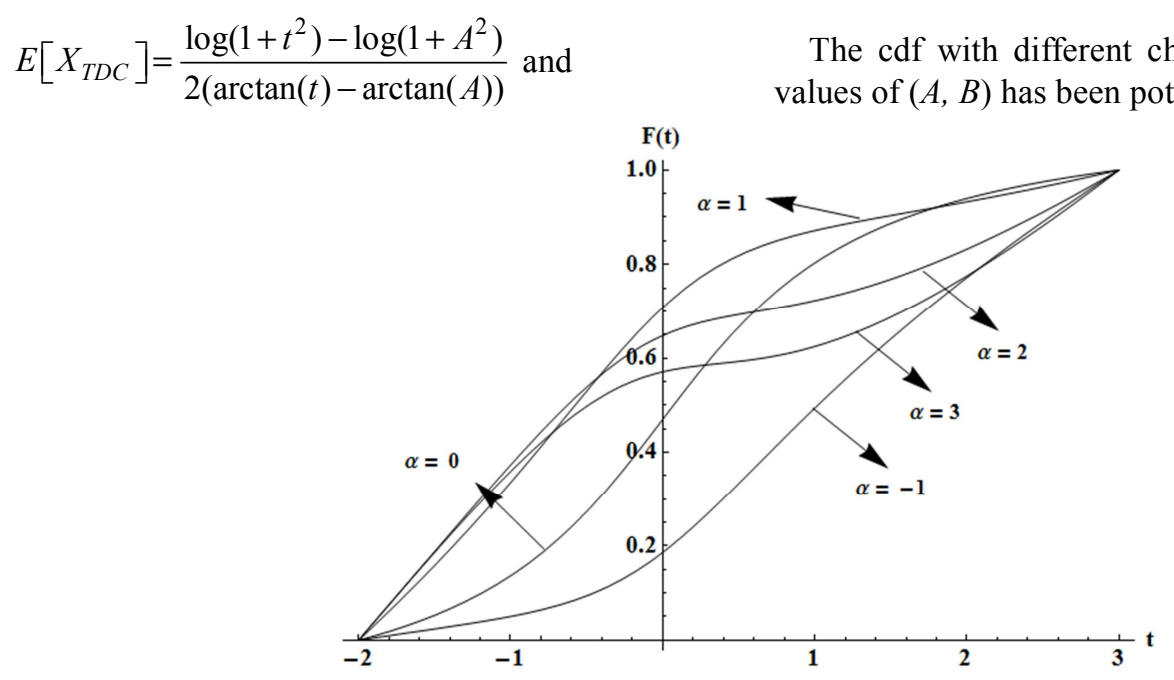

Figure 3. Shape of $c d f$ with different choices of parameter $\alpha$ and fixed $A=-2$ and $B=3$. 
Result 3: The $n^{\text {th }}$ order moment of ASTC distribution is given by

$$
E\left(X^{n}\right)=\frac{1}{D_{(A, B)}}\left[2\left\{I_{n}(B)-I_{n}(A)\right\}+\alpha^{2}\left\{I_{n+2}(B)-I_{n+2}(A)\right\}-2 \alpha\left\{I_{n+1}(B)-I_{n+1}(A)\right\}\right]
$$

Where, $I_{n}(Z)$ is the Gauss hypergeometric function (see [14], equation 3.194.5) and it can be written as $I_{n}(Z)=\int_{0}^{Z} \frac{y^{n}}{1+y^{2}} d y=\frac{Z^{n+1}}{n+1}{ }_{2} F_{1}\left(1, \frac{n+1}{2} ; \frac{n+3}{2} ;-Z^{2}\right)$

Proof: $E\left(X^{n}\right)=\frac{1}{D_{(A, B)}} \int_{A}^{B} \frac{x^{n}\left\{(1-\alpha x)^{2}+1\right\}}{\left(1+x^{2}\right)} d x=\frac{1}{D_{(A, B)}}\left[2 \int_{A}^{B} \frac{x^{n}}{\left(1+x^{2}\right)}-2 \alpha \int_{A}^{B} \frac{x^{n+1}}{\left(1+x^{2}\right)} d x+\alpha^{2} \int_{A}^{B} \frac{x^{n+2}}{\left(1+x^{2}\right)} d x\right]$

$$
=\frac{1}{D_{(A, B)}}\left[2 \int_{A}^{B} \frac{x^{n}}{\left(1+x^{2}\right)} d x-2 \alpha \int_{A}^{B} \frac{x^{n+1}}{\left(1+x^{2}\right)} d x+\alpha^{2} \int_{A}^{B} \frac{x^{n+2}}{\left(1+x^{2}\right)} d x\right]=\frac{1}{D_{(A, B)}}\left(2 G_{n}-2 \alpha G_{n+1}+\alpha^{2} G_{n+2}\right)
$$

Now, $G_{n}$ can be expressed as $G_{n}=\int_{0}^{B} \frac{x^{n}}{\left(1+x^{2}\right)} d x-\int_{0}^{A} \frac{x^{n}}{\left(1+x^{2}\right)} d x=I_{n}(B)-I_{n}(A)$.

Similarly, $G_{n+1}=I_{n+1}(B)-I_{n+1}(A)$ and $G_{n+2}=I_{n+2}(B)-I_{n+2}(A)$ and putting this results in (14) we get the desire result in equation (13).

In Particular,

$$
\begin{aligned}
E(X)= & \frac{1}{2 D}\left[(4 \alpha+2)[\arctan (B)-\arctan (A)]+\left(\alpha^{2}-1\right) \log \left[\frac{A^{2}+1}{B^{2}+1}\right]-\alpha(A-B)[\alpha(A+B)-4]\right] \\
E\left(X^{2}\right)= & \frac{1}{D}\left[(B-A)\{1-\alpha(B+A)\}+\frac{\alpha^{2}}{3}\left(3 A-3 B-A^{3}+B^{3}\right)+\alpha \log \left[\frac{B^{2}+1}{A^{2}+1}\right]+\alpha^{2}\{\arctan (B)-\arctan (A)\}\right] \\
E\left(X^{3}\right)= & \frac{1}{12 D}\left[\left(A^{2}-B^{2}\right)\left\{3 \alpha^{2}\left(A^{2}+B^{2}-2\right)-6\right\}+6\left(\alpha^{2}-1\right) \log \left[\frac{B^{2}+1}{A^{2}+1}\right]+\right. \\
& \left.(12-24 \alpha)\{\arctan (B)-\arctan (A)\}+8 \alpha\left(A^{3}-B^{3}+3 B-3 A\right)\right] \operatorname{and} \\
E\left(X^{4}\right)= & \frac{1}{D}\left[A-B+\frac{1}{3}\left(B^{3}-A^{3}\right)+\alpha\left(B^{2}-A^{2}\right)+\frac{\alpha}{2}\left(A^{4}-B^{4}\right)+\left(\alpha^{2}-2\right)[\arctan (A)-\arctan (B)]+\right. \\
& \left.\alpha^{2}\left\{B-A+\frac{1}{3}\left(A^{3}-B^{3}\right)-\frac{1}{5}\left(A^{5}-B^{5}\right)\right\}+\alpha \log \left[\frac{B^{2}+1}{A^{2}+1}\right]\right] .
\end{aligned}
$$

\section{Application in Real Life}

Though the Cauchy distribution has comparatively limited applications in real life due to non existence moment, but still it can be applied to model the data from many areas like mechanical and electrical theory, physical anthropology, measurement and calibration problem, depth map data, prices of speculative assets such as stock returns etc. [1]. In this an attempt has been made to present a data fitting illustration to compare the proposed distribution with some competitors. To this end the data about the relative difference of one year to next year of the log transformed exchange rate of the United
Kingdom Pound to the United States Dollar from 1800 to 2003 (for data set see [6]) has been considered. Alongside the proposed ASTC distribution (equation 10), skew Cauchy distribution in equation (4), truncated skew Cauchy distribution in equation (5), doubly truncated Cauchy distribution in equation (6), truncated skew Cauchy uniform distribution in equation (8). The MLE's of the parameters have been obtained by using numerical optimization routine. Note here that the MLE of the truncation limits $A$ and $B$ of ASTC distribution can be seen as $\hat{A}=X_{(1)}$ and $\hat{B}=X_{(n)}$ from the fact that the maximization of the likelihood w. r. t. $A$ or $B$ is equivalent to the minimization of the normalizing 
constant $D_{(A, B)}$ in the pdf in equation (10) w. r. t. $A$ or $B$. For the present data set the MLEs of $\mathrm{A}$ and $\mathrm{B}$ have been obtained as $\hat{A}=-0.606$ and $\hat{B}=1.481$. A similar argument is true also for estimators of the truncation limits of other truncated distribution considered for fitting here. While for the symmetrically truncated distributions the truncation limit has been chosen as $\hat{h}=X_{(n)}=1.481$. Akaike Information Criterion (AIC) and Bayesian Information Criterion (BIC) have been used for model comparison. In table 1 the MLEs of the parameters along with $\log$ likelihood $(\log L)$ AIC and $\mathrm{BIC}$ of the aforesaid distributions have been furnished.

Table 1. MLE, AIC, BIC and log-likelihood of Different Distributions.

\begin{tabular}{|c|c|c|c|c|c|c|}
\hline Distributions & $\hat{h}$ & $\hat{A}$, & $\hat{\boldsymbol{B}}$ & $\hat{\alpha}$ & AIC & BIC \\
\hline Skew Cauchy & $\begin{array}{ll}---- \\
--\end{array}$ & ----- & $\begin{array}{ll}---- \\
--\end{array}$ & 0.268 & 472.979 & 476.292 \\
\hline $\begin{array}{l}\text { Truncated skew } \\
\text { Cauchy }\end{array}$ & 1.481 & ----- & ----- & 0.187 & 282.133 & 288.759 \\
\hline $\begin{array}{l}\text { Doubly truncated } \\
\text { skew Cauchy }\end{array}$ & ----- & -0.606 & 1.481 & 0.112 & 318.207 & 328.147 \\
\hline $\begin{array}{l}\text { Truncated skew } \\
\text { Cauchy uniform }\end{array}$ & 1.481 & ---- & ---- & 0.577 & 282.227 & 288.253 \\
\hline ASTC & ----- & -0.606 & 1.481 & 0.763 & 138.393 & 148.333 \\
\hline
\end{tabular}

From the above results in table 1, the proposed distribution turns out to be the best choice for the exchange rate data of the United Kingdom Pound to the United States Dollar as compared to others in terms of both AIC and BIC scores.

\section{Conclusion}

A new alpha skew truncated Cauchy distribution has been introduced and some of its basic properties have been investigated. An application of data fitting with a real life data set has shown that the proposed distribution is far better as compared to the skew Cauchy distribution (equation 4), truncated skew Cauchy distribution (equation 5), two sided truncated Cauchy distribution (equation 6) and truncated skew Cauchy uniform distribution (equation 8). As such it may be concluded that the proposed distribution will be useful in modeling real life data.

\section{Acknowledgement}

Authors would like to express their sincere gratitude to the anonymous referees for reading the manuscript of the paper and fruitful comments.

\section{References}

[1] Johnson, N. L. and Kotz, S. (1970). Continuous Univariate Distributions, Vol. 1, John Wiley and Sons, New York.

[2] Nadarajah, S and Kotz, S. (2006). A Truncated Cauchy Distribution. International Journal of Mathemaical Education in Science and Technology. 37, 605-608.

[3] Chakraborty, S. and Sarmah, G. C. (2010). On Doubly Truncated Cauchy Distribution. Int. J. Agricult. Stat. Sci. 6 (2), 349-363.

[4] Azzalini, A. (1985). A class of distributions which includes the normal ones. Scand. J. Stat.. 12, 171 - 178.

[5] Chakraborty, S., Hazarika, P. J. (2011). A survey of the theoretical developments in univariate skew normal distributions, Assam Statistica Review. 25 (1), 41-63.

[6] Nadarajah, S, Kotz, S. (2007). A Skew Truncated Cauchy Distribution with application in economics. Applied Economics Letter. 14, 957-961.

[7] Huang, W. J., Chen, Y. H. (2007). Generalized skew Cauchy distribution, Statistics Probability Letters. 77, 1137 - 1147.

[8] Ashani, Z. N., Bakar, M. R. A., Ibrahim, N. A., Adam, M. B. (2016). A Skewed Truncated Cauchy Uniform Distribution and its Moment. Modern Applied Science. 10 (7), 174-182.

[9] Elal-Olivero, D. (2010). Alpha-skew-normal distribution. Proyecciones Journal of Mathematics. 29, 224 - 240.

[10] Harandi, S. S., Alamatsaz, M. H. (2013). Alpha-skew-Laplace distribution, Statist Statistics Probability Letters. 83 (3): 774 782 .

[11] Hazarika, P. J., Chakraborty, S. (2014). Alpha Skew Logistic Distribution, IOSR Journal of Mathematics. 10 (4) Ver. VI: 36-46.

[12] Hazarika, P. J., Chakraborty, S. (2015). A Note on Alpha Skew Generalized Logistic Distribution. IOSR Journal of Mathematics. 11 (1) Ver. IV: 85-88.

[13] Sharafi, M, Sajjadnia, Z, Behboodian, J. (2016). A new generalization of Alpha-Skew-Normal Distribution, Communications in Statistics-Theory and Methods. DOI: 10.1080/03610926.2015.1117639

[14] Prudnikov, A. P., Brychkov, Y. A., Marichev, O. I. (1986). Integrals and Series, Vols 1, 2 and 3 (Amsterdam: Gordon and Breach Science Publishers). 